\title{
A sensitive scale to assess nociceptive pain in patients with disorders of consciousness
}

\author{
Camille Chatelle, ${ }^{1,2}$ Steve Majerus, ${ }^{3}$ John Whyte, ${ }^{4}$ Steven Laureys, ${ }^{1,2}$ \\ Caroline Schnakers ${ }^{1,2,3}$
}

${ }^{1}$ Coma Science Group, Cyclotron Research Centre, University of Liège, Liège, Belgium ${ }^{2}$ Department of Neurology, University Hospital of Liège, Liège, Belgium

${ }^{3}$ Department of Cognitive Sciences, University of Liège, Liège, Belgium

${ }^{4}$ Moss Rehabilitation Research Institute, Philadelphia,

Pennsylvania, USA

Correspondence to Dr Caroline Schnakers, Coma Science Group, Cyclotron Research Centre, Sart Tilman, B30, University of Liège, 4000 Liège, Belgium;

c.schnakers@ulg.ac.be

Received 16 April 2012

Revised 23 July 2012

Accepted 1 August 2012

Published Online First

20 August 2012

\section{ABSTRACT}

Objective To determine the sensitivity of the Nociception Coma Scale (NCS), the first scale developed to assess nociceptive pain in vegetative state and minimally conscious state patients, in comparing behavioural changes in response to noxious versus non-noxious stimulation.

Methods The NCS was administered to assess patients' responses in three conditions: (1) baseline (observation of spontaneous behaviours), (2) non-noxious/tactile stimulation (taps on the patient's shoulder), and (3) noxious stimulation (pressure on the nail bed).

Results We included 64 patients (27 vegetative state and 37 minimally conscious state; age range 20-82 years; 22 traumatic brain injury; 21 in the acute stage). The NCS total scores and subscores (motor, verbal and facial) were higher for the noxious versus the nonnoxious stimulation conditions. We did not observe a difference between the non-noxious and the noxious stimulation conditions for the visual subscale. We also found a NCS cut-off value of 4 differentiating the patients receiving a noxious stimulation from patients receiving a non-noxious stimulation. The exclusion of the visual subscale increased the cut-off sensitivity (from $46 \%$ to $73 \%$; specificity of $97 \%$ and accuracy of $85 \%$ ).

Conclusion We propose a new version of the NCS excluding the visual subscale, the NCS-R, which constitutes a highly sensitive tool to assess responses to nociceptive pain in severely brain injured patients.

Detecting pain in non-communicative patients with disorders of consciousness constitutes a real challenge. ${ }^{1}$ Schnakers et al developed the Nociception Coma Scale (NCS) ${ }^{2}$ based on pain scales for non-communicative patients with advanced dementia $^{4}$ or newborns ${ }^{5}$ to assess behaviours linked to nociceptive pain in the vegetative state ${ }^{6}$ (VS) (preserved arousal but no behavioural signs of consciousness) and in the minimally conscious state $(M C S)^{7}$ (preserved arousal and reproducible but fluctuating behavioural signs of consciousness). In the validation study, in addition to good interrater agreement and good concurrent validity with existing pain scales designed for non-communicative patients, ${ }^{8-11}$ the NCS was the only scale to show a significant difference as a function of the consciousness level suggesting the NCS is highly sensitive to differentiate behavioural pattern in VS versus MCS patients. A noxious stimulus (pressure applied to the nail bed) was used in order to ensure that the behavioural changes observed were directly linked to the stimulation. ${ }^{2}$ However, this does not ensure that these behavioural changes are actually specific to noxious stimulation, as they may also occur spontaneously or in response to non-noxious stimulation. Further investigation has therefore to be performed in order to ensure that the NCS is particularly sensitive to noxious stimulation. The objective of the present study was to compare the behavioural changes observed with the NCS in response to noxious versus non-noxious stimuli in order to determine its sensitivity in assessing behaviours linked to nociceptive pain in severely brain-injured patients.

\section{METHODS}

\section{Participants}

This study was a prospective multicentre study including patients from the intensive care and the neurology units of the university hospital of Liège as well as from the neurorehabilitation centres and nursing homes that are part of the Belgian federal network for vegetative and minimally conscious states. Inclusion criteria were: (1) age $\geq 18$ years; (2) no administration of neuromuscular blockers or sedation within the $24 \mathrm{~h}$ of enrolment; (3) the presence of periods of eye opening (indicating wakefulness and rest cycles); and (4) a diagnosis of VS or MCS, based on behavioural assessment performed using the Coma Recovery Scale-Revised. ${ }^{12}$ Exclusion criteria were: (1) documented history of prior brain injury; (2) premorbid history of developmental, psychiatric or neurologic illness resulting in documented functional disability up to time of the injury; and (3) upper limb contusions, fractures or flaccid paralysis. The study was approved by the Ethics Committee of the Faculty of Medicine of the University of Liège and written informed consent was obtained by the patient's legal representative.

\section{Procedure}

The NCS consists of four subscales assessing motor, verbal, visual and facial responses; each subscore ranges from 0 to 3 (total score: $0-12$ ) (box 1). The scale was administered to assess patients' responses in three different conditions: (1) baseline, (2) nonnoxious stimulation, and (3) noxious stimulation. We included a baseline condition in order to be sure that the NCS total score obtained in response to stimulation is different from what is obtained spontaneously. We also included a non-noxious/ tactile stimulation condition as somatosensory and noxious stimulations are the closest classes of stimulation we could compare. During the first 


\section{Box 1 Protocol of the Nociception Coma Scale}

\section{Motor response}

3 - Localisation to painful stimulation

2 - Flexion withdrawal

1 - Abnormal posturing

0 - None/flaccid

\section{Verbal response}

3 - Verbalisation (intelligible)

2 - Vocalisation

1 - Groaning

0 - None

\section{Visual response}

3 - Fixation

2 - Eye movements

1 - Startle

0 - None

\section{Facial expression}

3 - Cry

2 - Grimace

1 - Oral reflexive movement/startle response

0 - None

condition (ie, baseline), we observed the patient's spontaneous behaviours during $60 \mathrm{~s}$ (as used in ${ }^{12}$ ). During the second condition (ie, non-noxious/tactile stimulation), we applied five rapid taps on the top of the patient's right and left shoulder. ${ }^{13}$ Finally, during the third condition (ie, noxious stimulation), we applied pressure on the nail bed (as used in ${ }^{12}$ and $^{14}$ ) of the middle finger of the right and left hand using a Newton-meter (Force Dial, FDN 200 model; Connecticut, USA; http://www.wagnerinstruments.com) which allows the examiner to gauge the amount of pressure applied to the patient. ${ }^{2}$ The fingernail pressure increased progressively, was administered for a minimum of $5 \mathrm{~s}^{12}$ and was stopped as soon as a behavioural response was observed.

Behavioural responses were recorded for a period of $10 \mathrm{~s}$ (as used in Giacino et $a l^{12}$ ) after each stimulation (ie, non-noxious and noxious). The best responses (ie, the highest score) obtained across right and left side stimulation were scored. In order to ensure a sufficient level of arousal, each condition was administered while patients showed spontaneous eye opening. The entire procedure lasted less than $5 \mathrm{~min}$. Patients' consciousness level was randomly assessed before or after this procedure by using the Coma Recovery Scale-Revised (CRS-R). The CRS-R consists of 23 hierarchically arranged items that comprise six subscales addressing arousal, auditory, visual, motor, oromotor/ verbal and communication functions. The lowest item on each subscale represents reflexive activity while the highest item represents cognitively-mediated behaviours. ${ }^{12}$ We did not apply the additional noxious stimulation requested in the motor subscale of the CRS-R but instead scored this item on the basis of the experimental noxious stimulation condition already applied.

\section{Statistical analyses}

As our data (ie, NCS total scores and subscores) were not normally distributed according to Shapiro-Wilk tests $(W<1)$, we decided to use non-parametrical statistical analyses. We have used a Friedman ANOVA with repeated measures on condition (ie, baseline vs non-noxious vs noxious) in order to detect differences in NCS total scores and subscores (ie, motor, visual, verbal and facial). We further investigated significant differences $(p<0.01$; Bonferroni corrected $p$ value threshold) between the different conditions with the Wilcoxon signed ranks test $(p<0.017$; Bonferroni corrected $p$ value threshold $)$.

Using a receiver operating characteristic (ROC) analysis, we also determined an NCS cut-off value differentiating the patients receiving a noxious stimulation from patients receiving a non-noxious stimulation. We assessed the discriminatory power of this threshold as regards its sensitivity (ie, the true positive rate or the proportion of patients who have received noxious stimulation and have an NCS total score above a determined cut-off) and its specificity (ie, the true negative rate or the proportion of patients who have received non-noxious stimulation and have a NCS total score below a determined cut-off).

\section{RESULTS}

We included 64 patients, of whom 27 were VS and 37 MCS according to the behavioural assessment performed using the CRS- $\mathrm{R}^{12}$ (age range 20-82 years; 24 women). Aetiology was traumatic $(n=22)$, post-anoxic $(n=20)$, intracerebral haemorrhage $(n=8)$, metabolic $(n=4)$, ischaemic stroke $(n=3)$, encephalitis $(n=3)$ and others $(n=4)$. Twenty-one patients were assessed in the acute stage (ie, $<1$ month post-injury) and 43 in the chronic stage (interval ranging from 1 month to 6 years). According to a Mann-Whitney $U$ test we performed, the amount of pressure that was applied $\left(52.77 \pm 10.39 \mathrm{~N} / \mathrm{cm}^{2}\right)$ in the noxious stimulation condition was not different according to the diagnosis (VS vs MCS) $(\mathrm{U}=421.5, \mathrm{p}=0.51)$.

Using a Friedman ANOVA with repeated measures on condition (ie, baseline vs non-noxious vs noxious), we obtained significant group effects for the total scores as well as for all subscores (table 1). Additional analyses using Wilcoxon signed ranks tests revealed a difference between baseline and noxious stimulation conditions as well as between non-noxious and noxious stimulation conditions for the total scores and for the motor, verbal and facial subscores. For the visual subscores, we obtained a difference between the baseline and both nonnoxious and noxious stimulation conditions, but no difference between non-noxious and noxious stimulation conditions (table 2 and figure 1). As regards these results, we performed $\chi^{2}$ tests to verify whether each of the behaviours included in the visual subscale (ie, startle, eye movement and fixation) were not observed more frequently in the noxious versus the non-noxious stimulation condition. We did not find any difference ( $p>0.05)$ for each of these behaviours, suggesting they are equally observed in both conditions.

Table 1 Friedman analysis of variance with repeated measures on condition (ie, baseline vs non-noxious vs noxious)

\begin{tabular}{llllll}
\hline NCS & Baseline & Non-noxious & Noxious & $\chi^{2}$ & p Value \\
\hline Total & $0.92 \pm 1.19$ & $1.48 \pm 1.37$ & $4.77 \pm 1.84$ & 101.46 & $<0.0001^{*}$ \\
Motor & $0.14 \pm 0.35$ & $0.21 \pm 0.45$ & $1.63 \pm 0.83$ & 96.35 & $<0.0001^{*}$ \\
Verbal & $0.09 \pm 0.39$ & $0.06 \pm 0.30$ & $0.37 \pm 0.70$ & 20.28 & $<0.0001^{*}$ \\
Visual & $0.34 \pm 0.82$ & $0.98 \pm 1.05$ & $1.25 \pm 0.97$ & 47.41 & $<0.0001^{*}$ \\
Facial & $0.34 \pm 0.54$ & $0.25 \pm 0.51$ & $1.43 \pm 0.82$ & 73.48 & $<0.0001^{*}$ \\
\hline
\end{tabular}

Asterisk marks significant difference $\left({ }^{*} p<0.01\right.$; Bonferroni corrected $\mathrm{p}$ value threshold). NCS, Nociception Coma Scale. 
Table 2 Wilcoxon signed ranks tests comparing conditions between each other

\begin{tabular}{lllllllll}
\hline & \multicolumn{2}{l}{$\begin{array}{l}\text { Baseline vs non- } \\
\text { noxious }\end{array}$} & & \multicolumn{2}{l}{$\begin{array}{l}\text { Baseline vs } \\
\text { noxious }\end{array}$} & & \multicolumn{2}{l}{$\begin{array}{l}\text { Non-noxious vs } \\
\text { noxious }\end{array}$} \\
\cline { 2 - 3 } NCS & $\mathbf{Z}$ & $\mathbf{p}$ Value & & $\mathbf{Z}$ & $\mathbf{p}$ Value & & $\mathbf{Z}$ & p Value \\
\hline Total & 3.13 & $<0.002^{*}$ & & 7.49 & $<0.0001^{*}$ & & 7.49 & $<0.0001^{*}$ \\
Motor & 1.15 & 0.24 & & 7.01 & $<0.0001^{*}$ & & 6.78 & $<0.0001^{*}$ \\
Verbal & 0 & 1 & & 2.77 & $0.006^{*}$ & & 3.33 & $<0.001^{*}$ \\
Visual & 4.63 & $<0.0001^{*}$ & & 5.58 & $<0.0001^{*}$ & & 2.24 & 0.02 \\
Facial & 1.58 & 0.11 & & 6.41 & $<0.0001^{*}$ & & 5.93 & $<0.0001^{*}$ \\
\hline
\end{tabular}

Asterisk marks significant difference ( ${ }^{*} p<0.017$; Bonferroni corrected $p$ value threshold). NCS, Nociception Coma Scale.

Using a ROC analysis, we identified an NCS cut-off value of 4 differentiating the patients receiving a noxious stimulation from patients receiving a non-noxious stimulation, with a sensitivity of $46 \%$ and a specificity of $97 \%$ (accuracy of $72 \%$ ), all consciousness levels confounded. We decided to perform the same analysis without the visual subscale. We also obtained a cut-off value of four but with a sensitivity of $73 \%$ and a specificity of $97 \%$ (accuracy of $85 \%$ ). The same cut-off value was found for the MCS group, with a sensitivity of $83 \%$ and a specificity of $95 \%$ (accuracy of $89 \%$ ). We obtained a cut-off value of 3 for the VS group, with a sensitivity of $96 \%$ and a specificity of $89 \%$ (accuracy of $93 \%$ ).

In the light of these results, we decided to test a revised version of the NCS including the motor, verbal and facial subscores and excluding the visual subscore. We used the Mann-Whitney $U$ test to assess whether the total scores of the revised version were different according to the diagnosis (VS vs $\mathrm{MCS}$ ), the onset (acute vs chronic) and the aetiology (traumatic vs non-traumatic) $(p<0.017$; Bonferroni corrected $p$ value threshold). We found a significant difference as a function of the diagnosis $(U=219, p<0.001)$. No significant difference was observed as either a function of the aetiology $(U=405, p=0.42)$ or the onset ( $U=374, p=0.27)$. Using a Friedman ANOVA with repeated measures on experimental condition (ie, baseline vs non-noxious vs noxious), we obtained significant effects of experimental condition for the total scores $\left(\chi^{2}=106,39\right.$,

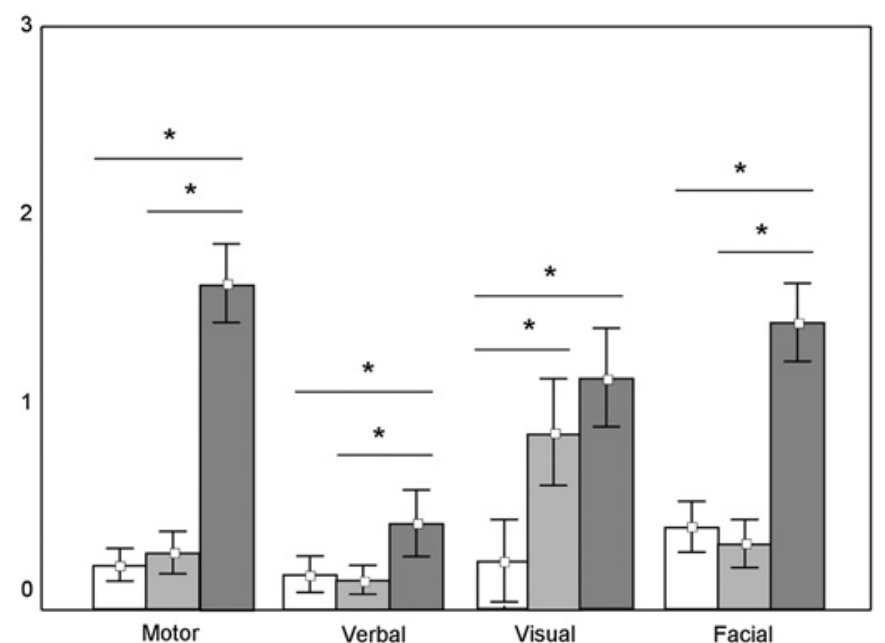

Figure 1 Mean (and SD) of Nociception Coma Scale subscores (0-3) in baseline (white), non-noxious stimulation (grey) and noxious stimulation (dark grey) conditions. Asterisk marks significant difference between conditions $\left({ }^{*} p<0.017\right.$; Bonferroni corrected $p$ value threshold). $\mathrm{p}<0.001)$. Additional analyses using Wilcoxon signed ranks tests revealed a difference between baseline and noxious stimulation conditions $(Z=7.36, p<0.001)$ as well as between non-noxious and noxious stimulation conditions $(Z=7.62, p<0.001)$ for the total scores (figure 2).

\section{DISCUSSION}

The objective of the present study was to compare the behavioural changes observed with the NCS in noxious as well as non-noxious stimulation conditions in order to determine its sensitivity to assess behaviours linked to nociceptive pain in severely brain-injured patients.

According to our results, the total scores obtained in the nonnoxious stimulation condition were not significantly different from those obtained in the baseline condition, suggesting the NCS is not sensitive for assessing responses to non-noxious stimulation. On the contrary, the NCS total scores were higher for the noxious stimulation condition as compared to both baseline and non-noxious stimulation conditions, suggesting the NCS is sensitive for assessing responses to noxious stimulation.

As regards the subscales, the motor, verbal and facial subscores showed similar results. However, we did not observe a difference between the non-noxious and the noxious stimulation conditions for the visual subscores. More specifically, the behaviours included in this subscale were observed as frequently in response to non-noxious stimulation as in response to noxious stimulation. Finally, according to the ROC curve analysis we performed, the exclusion of this subscale increased the sensitivity of the cutoff score of 4 from $46 \%$ to $73 \%$. In other words, the visual subscale does not seem to be sensitive for assessing responses to noxious stimulation. On the contrary, its exclusion seems to increase the sensitivity of the scale.

We therefore propose a revised version of the Nociception Coma Scale (or NCS-R), including the motor, verbal and facial subscores and excluding the visual subscore. Like its original version, ${ }^{2}{ }^{3}$ the NCS-R total score differs according to the diagnosis, higher total scores being observed in MCS versus VS patients. In parallel, the threshold differentiating the NCS-R total score obtained in the noxious versus the non-noxious stimulation condition varied according to the diagnosis, with a cut-off of 4 for MCS patients and 3 for VS patients. The lower threshold observed for VS patients has to be related to the lower NCS-R total scores obtained in response to noxious stimulations (range 1-4) as compared to MCS patients (range 2-8). Indeed, VS patients typically manifest less complex behaviours (eg, abnormal posturing, groaning and oral reflexive movements) as compared MCS patients (eg, localisation to noxious stimulation, intelligible verbalisations and grimaces), ${ }^{1}$ leading to lower NCS$\mathrm{R}$ total scores in VS patients. The fact that VS patients present less complex behaviours as compared to MCS patients may be explained by previous neuroimaging studies. Brain activation to nociceptive pain in VS patients seems to involve non-integrated low-level processing (ie, the midbrain as well as the thalamus and the primary somatosensory cortex, which are usually related to nociception - the neural processes of encoding and processing noxious stimuli (ie, an actually or potentially tissue damaging event)' - and, only partly, to basic sensory-discriminative aspects of pain processing), ${ }^{15-18}$ whereas brain activation in MCS patients seems to involve integrated high-level processing (ie, the thalamus and the primary somatosensory cortex connected to the secondary somatosensory cortex, which are usually related to the sensory-discriminative aspects of pain processing but also the anterior cingulate, insular and prefrontal cortices 
Figure 2 Mean and SD (upper panel) as well as dispersion (lower panels) of the Nociception Coma Scale-Revised (NCS-R) total scores in baseline (white), non-noxious stimulation (grey) and noxious stimulation (dark grey) conditions for each group: vegetative state (VS) and minimally conscious state (MCS). Asterisk marks significant difference between groups and conditions $\left({ }^{*} p<0.017\right.$; Bonferroni corrected $\mathrm{p}$ value threshold).

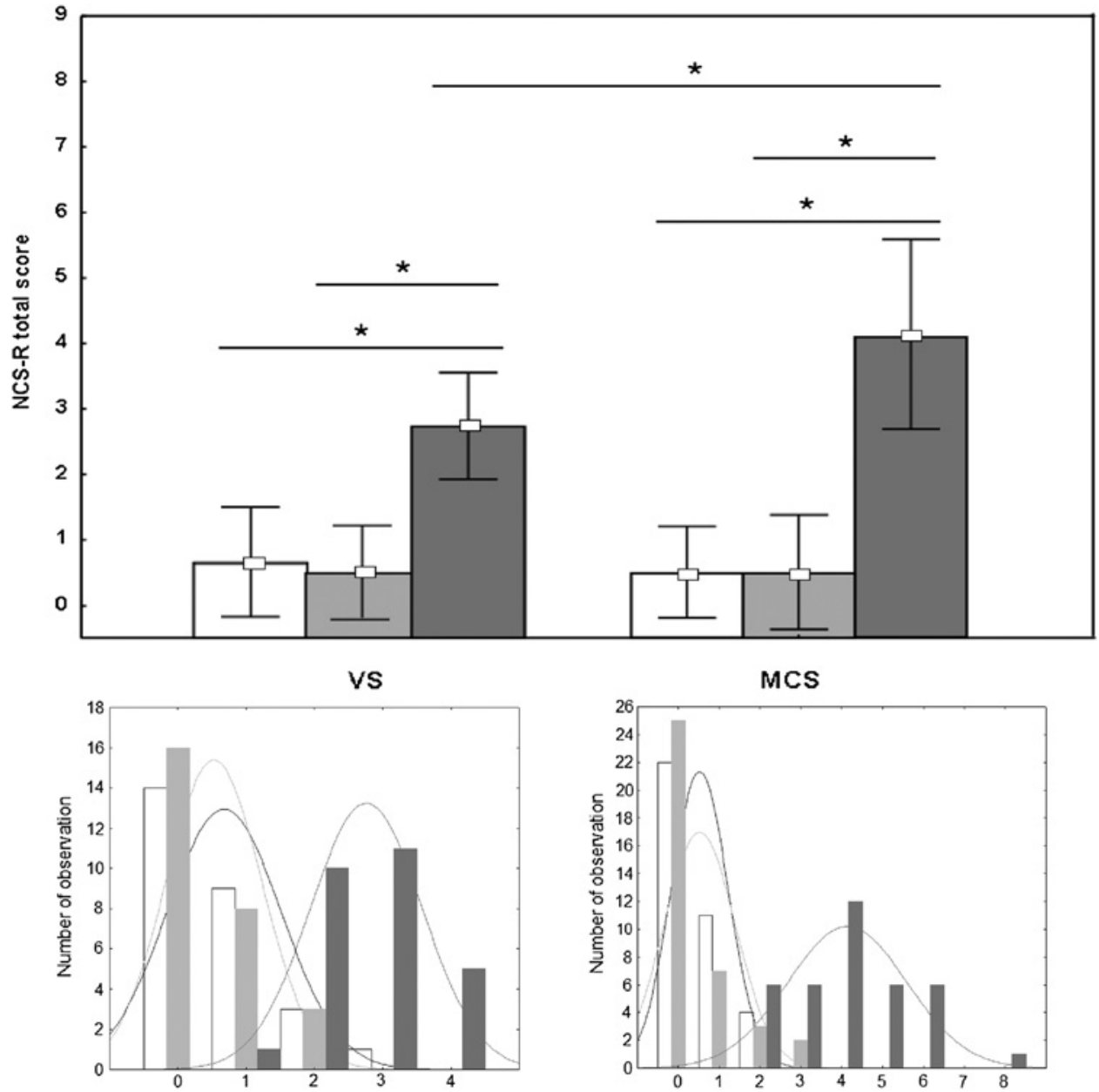

which have been related to pain-related affective and cognitive behaviours). ${ }^{16} 18-20$

It is therefore not surprising that we observed a difference in the behavioural responses of these patients and hence in the cutoff score. In fact, no VS patient had a score above 4 in response to noxious stimulation, suggesting that this threshold may potentially reflect the presence of integrated high-level pain processing in severely brain-injured patients. Future neuroimaging studies will have to confirm that a score above 4 is related to such processing and, more precisely, to brain areas related to sensory discriminative systems (ie, primary and secondary somatosensory cortex) as well as motivationalaffective and cognitive-evaluative systems (ie, cingulate, insula and prefrontal cortices). ${ }^{18} 20$ Even though we found a difference between the NCS-R total scores obtained in VS and MCS patients, the primary aim of the scale is to assess behavioural responses to nociceptive pain and not to assess the patients' consciousness level as other sensitive scales can be used for this purpose. ${ }^{12}$ Finally, we did not obtain differences according to the onset or the aetiology, suggesting that this scale can be used in the acute or chronic setting as well as with traumatic or nontraumatic patients. One could argue that the psychometric data of this version were not tested and that the NCS-R could be a sensitive but not valid tool. Nevertheless, in our previous study, the verbal, visual and motor subscales showed significant correlations and hence good concurrent validity with pain scales for non-communicative patients such as patients with dementia and newborns. The inter-rater agreement was also good to excellent for each of these subscales. ${ }^{2}$ These results suggest that the NCS-R, which includes these subscales, constitutes a sensitive and validated tool to assess behaviours linked to nociceptive pain in patients with disorders of consciousness.

This study has nevertheless several limitations. First, we used a fixed order for the administration of the conditions (ie, baseline, non-noxious and noxious stimulation conditions). We decided to apply the baseline and non-noxious conditions before the noxious one since noxious stimulations have a longer lasting effect than non-noxious ones. If the noxious stimulation is applied first, it is likely that the stimulation will affect the responses observed in the other conditions, particularly as the time window between conditions is short (ie, 20-60 s). Second, the duration of the baseline and of both the non-noxious and noxious stimulation conditions was different. We decided to use a $60 \mathrm{~s}$ baseline in order to use the same procedure as that is used in standardised scales such as the CRS-R. ${ }^{12}$ Since the duration of observation is longer, more behaviours and therefore higher total scores (and subscores) might have been observed during this condition. This could have diminished our chance to obtain a difference between this condition and the other ones (ie, nonnoxious and noxious). Despite this assumption, we still obtained significant differences in the NCS/NCS-R total scores and subscores between the baseline and the noxious stimulation condition. Finally, as our patients are non-communicative, we cannot exclude that some of them were experiencing pain in response to non-noxious stimulation (even if we excluded patients with documented upper limb contusions or fractures). However, if this had been the case for a significant portion of our population, the NCS/NCS-R total scores would have been as 
high in the non-noxious stimulation condition as in the noxious stimulation condition, which was not the case.

\section{CONCLUSION}

Our results suggest that the revised version of the NCS (NCS-R) is a highly sensitive tool to assess responses to noxious stimulation in severely brain injured patients, and is more sensitive than the original version of the NCS. The potential relation between an NCS-R cut-off value of 4 and pain processing should however be further investigated in the future. Results showing a relationship between the NCS-R total score and integrated high-level pain processing at a neural level would further support the use of such a scale for detecting and treating pain in patients with disorders of consciousness. In parallel, studies assessing the clinical utility of the scale are warranted. Until now, we used experimental nociceptive pain (ie, mechanicalpressure on the fingernail). Research showing a decrease of the NCS-R total scores after antalgic treatment as compared before treatment in patients known to have painful conditions (eg, fractures, soft tissue injuries or severe spasticity) would confirm the clinical utility of the NCS-R.

Overall, the NCS-R constitutes a promising clinical tool for assessing pain in severely brain-injured patients, and hence may lead to significant improvement of the care provided to these patients in the future.

Contributors CC: collection, analysis and interpretation of the data, drafting the manuscript. SM, SL: revising the manuscript. JW: conceptualisation of the study and revising the manuscript. SC: design of the study, analysis and interpretation of the data, drafting the manuscript. CS and CC conducted the statistical analysis.

Funding This study was supported by the Belgian Federal Public Health, the National Funds for Scientific Research (FNRS), James S. McDonnell Foundation, Mind Science Foundation, European Commission (Mindbridge, DISCOS, DECODER \& COST), Concerted Research Action (ARC 06/11-340), Public Utility Foundation 'Université Européenne du Travail', 'Fondazione Europea di Ricerca Biomedica' and the University of Liège. CS is postdoctoral researcher, CC is research fellow, and SM and SL are senior research associates at FNRS.

Competing interests None.

Ethics approval Ethics Committee of the Faculty of Medicine of the University of Liège.

Provenance and peer review Not commissioned; externally peer reviewed.

\section{REFERENCES}

1. Schnakers C, Zasler ND. Pain assessment and management in disorders of consciousness. Curr Opin Neurol 2007;20:620-6.

2. Schnakers C, Chatelle C, Vanhaudenhuyse A, et al. The Nociception Coma Scale: a new tool to assess nociception in disorders of consciousness. Pain 2010;148:215-19.

3. Schnakers C, Chatelle C, Majerus S, et al. Assessment and detection of pain in non-communicative severely brain-injured patients. Expert Rev Neurother 2010;10:1725-31.

4. Hummel P, van Dijk M. Pain assessment: current status and challenges. Semin Fetal Neonatal Med 2006;11:237-45.

5. Anon. The management of persistent pain in older persons. J Am Geriatr Soc 2002; 50:205-24.

6. The Multi-Society Task Force on PVS. Medical aspects of the persistent vegetative state (1). N Engl J Med 1994;330:1499-508.

7. Giacino J, Ashwal S, Childs $\mathrm{N}$, et al. The minimally conscious state: definition and diagnostic criteria. Neurology 2002;58:349-53.

8. Lawrence J, Alcock D, McGrath P, et al. The development of a tool to assess neonatal pain. Neonatal Netw 1993;12:59-66

9. Merkel SI, Voepel-Lewis T, Shayevitz JR, et al. The FLACC: a behavioral scale for scoring postoperative pain in young children. Pediatr Nurs 1997;23:293-7.

10. Warden V, Hurley AC, Volicer L. Development and psychometric evaluation of the Pain Assessment in Advanced Dementia (PAINAD) scale. J Am Med Dir Assoc 2003:4:9-15.

11. Feldt KS. The checklist of nonverbal pain indicators (CNPI). Pain Manag Nurs 2000;1:13-21.

12. Giacino J, Kalmar K, Whyte J. The JFK Coma Recovery Scale-Revised: measurement characteristics and diagnostic utility. Arch Phys Med Rehabil 2004;85:2020-9.

13. Gill-Thwaites H, Munday R. The sensory modality assessment and rehabilitation technique (SMART): a valid and reliable assessment for vegetative state and minimally conscious state patients. Brain Inj 2004;18:1255-69.

14. Teasdale G, Jennett B. Assessment of coma and impaired consciousness: a practical scale. Lancet 1974;2:81-4.

15. Laureys $\mathbf{S}$, Faymonville ME, Peigneux $\mathrm{P}$, et al. Cortical processing of noxious somatosensory stimuli in the persistent vegetative state. Neuroimage 2002;17:732-41.

16. Boly M, Faymonville ME, Peigneux $\mathrm{P}$, et al. Cerebral processing of auditory and noxious stimuli in severely brain injured patients: differences between VS and MCS Neuropsychol Rehabil 2005;15:283-9.

17. Loeser JD, Treede RD. The Kyoto protocol of IASP basic pain terminology. Pain 2008; 137:473-7

18. IASP. Classification of Chronic Pain: Descriptions of Chronic Pain Syndromes and Definitions of Pain Terms. Task Force on Taxonomy, Vol. Supp/ 3. Seatle: IASP Press, 1994.

19. Boly M, Faymonville ME, Schnakers C, et al. Perception of pain in the minimally conscious state with PET activation: an observational study. Lancet Neurol 2008; 7:1013-20.

20. Kupers $\mathbf{R}$, Kehlet $\mathrm{H}$. Brain imaging of clinical pain states: a critical review and strategies for future studies. Lancet Neurol 2006;5:1033-44. 


\section{A sensitive scale to assess nociceptive pain in patients with disorders of consciousness}

Camille Chatelle, Steve Majerus, John Whyte, et al.

J Neurol Neurosurg Psychiatry 2012 83: 1233-1237 originally published online August 20, 2012

doi: 10.1136/jnnp-2012-302987

Updated information and services can be found at:

http://jnnp.bmj.com/content/83/12/1233.full.html

These include:

References This article cites 19 articles, 1 of which can be accessed free at: http://jnnp.bmj.com/content/83/12/1233.full.html\#ref-list-1

Email alerting Receive free email alerts when new articles cite this article. Sign up in service the box at the top right corner of the online article.

Notes

To request permissions go to:

http://group.bmj.com/group/rights-licensing/permissions

To order reprints go to:

http://journals.bmj.com/cgi/reprintform

To subscribe to BMJ go to:

http://group.bmj.com/subscribe/ 